\title{
There May Yet Be Non-Causal Explanations (of Particular Events)
}

Penultimate draft - forthcoming in Fournal for General Philosophy of Science

\begin{abstract}
There are many putative counterexamples to the view that all scientific explanations are causal explanations. Using a new theory of what it is to be a causal explanation, Bradford Skow has recently argued that several of the putative counterexamples fail to be non-causal. This paper defends some of the counterexamples by showing how Skow's argument relies on an overly permissive theory of causal explanations.
\end{abstract}

Keywords: causal explanation; partial explanation; the barometer; causal histories.

\section{Introduction}

No one denies that many explanations are causal. However, since Salmon (1984) and Lewis (1986a), not many philosophers have defended the view that all explanations are causal explanations. This is largely due to a host of putative counterexamples presented over the past decades. Using a new theory of what it is to be a causal explanation, Bradford Skow (2014) 
has recently argued that several of the putative counterexamples fail to be non-causal. On this basis, Skow defends the view that all explanations of particular events are causal explanations, except for those that are what he calls in-virtue-of explanations. ${ }^{1}$

One way to argue against Skow is to show that some genuine scientific explanation does not count as a causal explanation on his theory. Another way is to argue that Skow's theory of causal explanations is false. I do the latter. More precisely, I argue that the theory of causal explanations to which Skow appeals is overly permissive - indeed, so permissive as to count obvious non-explanations as causal explanations. I also suggest a natural improvement of Skow's theory, and argue that on this modified theory there is at least one class of counterexamples to the view that all explanations are causal. This serves to further undermine the prospects for arguing that all explanations are causal.

\section{Skow's Theory of Causal Explanations}

In arguing against the putative counterexamples to the view that all explanations are causal, Skow does not appeal to our pre-theoretic judgments about which explanations seem causal and non-causal. This should not be surprising, since the putative counterexamples have so far struck most philosophers as involving non-causal explanation. Instead, Skow uses a particular

\footnotetext{
${ }^{1}$ Skow says that in-virtue-of explanations explain why some facts obtain by appealing to "deeper" facts that ground them. To illustrate, Skow notes that a scientist may explain why a piece of glass is fragile by citing its molecular structure. Such an explanation would be an in-virtue-of explanation, since the molecular structure grounds its fragility. (Skow 2014, 446-447) Since I will not be discussing in-virtue-of explanations in what follows, I will for convenience use "explanation" in a restricted sense as referring only to explanations that are not in-virtue-of explanations (although see footnote 8).
} 
theory of causal explanation. Skow works up to his theory in stages, but it suffices for our purposes to examine the end-result:

STCE: "A body of fact partially causally explains $\mathrm{E}$ iff it is a body of fact about what causes, if any, E had; or [...] it is a body of fact about what it would have taken for some specific alternative or range of alternatives to E to have occurred instead." (Skow, 2014: 449)²

Two clarifications of this theory are in order: First, since Skow argues only that all explanations of particular events are causal, STCE should similarly be restricted to explanations in which the explanandum E is a particular event. Second, STCE is meant to allow for bodies of fact to partially causally explain E even when those bodies of facts merely rule out some possible causes of $\mathrm{E}$ without narrowing them down to a single cause. Thus "about" in STCE is understood in a broad sense in which something can be about $\mathrm{X}$ merely by ruling out some ways in which $\mathrm{X}$ might be.

To illustrate, let us see how this deals with one putative counterexample due to Nerlich (1979). Suppose a cloud of dust particles is moving through space. There are no forces acting on the particles, so they travel in "straightest" lines, i.e. along the shortest possible paths between their initial and final positions. But because space is curved the shape of the cloud keeps changing as it moves between different points in space (it might be shorter for some of

${ }^{2}$ As Skow indicates, the first part of this theory is very similar, if not identical, to that proposed by Lewis (1986a). Thus Skow's theory is even more permissive than Lewis's already quite permissive theory of causal explanations. That said, my argument against Skow (presented in section 3) is not only that the addition of the second part of the theory makes it too permissive, but that the first part is already too permissive (because it counts obvious non-explanations as partial causal explanations). So if my argument succeeds against Skow's theory, it also succeeds against Lewis's. 
the particles to move around a bump in space, for example, rather than going over it). Nerlich takes this to be a counterexample to the thesis that all explanations of particular events are causal explanations, roughly because the curvature of space is not a cause of the cloud's changing shape. In reply, Skow points out that Nerlich's explanation "rules out all possible causal histories in which free particles do not travel on straightest lines but instead require external causes to do so." (Skow, 2014: 451) So although Nerlich's explanation does not specify a cause of the cloud's changing shape, it does partially causally explain it in virtue of ruling out certain possible causal histories. ${ }^{3}$

It is worth noting that the view that Skow is actually defending here and elsewhere in his paper is considerably weaker than the view that all explanations are causal explanations. It is, rather, that all explanations are partial causal explanations. Indeed, as STCE makes evident, Skow does not even give a theory of complete causal explanations. This poses at least a prima facie problem for Skow since unless the stronger claim can be defended, it seems that there is explanatory information that is not causal. And that in turn undermines at least the spirit, if not the letter, of the view that all explanations are causal. However, I shall not discuss this problem here and instead argue that Skow has not even successfully defended the claim that all explanations are partially causal.

\footnotetext{
${ }^{3}$ One might wonder about the kind of possibility at play in Skow's view: Need a partial causal explanation rule our causal histories that are physically possible or is it enough for them to rule out causal histories that are metaphysically, epistemically, or even just logically possible? Although Skow does not address this question explicitly, it is clear from his response to Nerlich's example that ruling out physically possible causal histories is not required. After all, worlds in which free particles do not travel on straightest lines are physically impossible, so ruling out causal histories in which that happens is not to rule out physically possible causal histories. Thus we see that the kind of possibility in play must be metaphysical, epistemic, or logical possibility.
} 


\section{An Objection to Skow's Theory}

This section presents an objection to Skow's theory of causal explanations, STCE. The objection is based on a standard counterexample to Hempel's (1965) DN-model of scientific explanations, viz. the "explanation" of a storm in terms of a barometer reading. Salmon describes the case as follows:

The barometer. If a sharp drop in the reading on a properly functioning barometer occurs, we can infer that there will be a storm - for the sake of argument, let us assume that there is a law that whenever the barometric pressure drops sharply a storm will occur. Nevertheless, we do not want to say that the barometric reading explains the storm, since both the drop in barometric reading and the occurrence of the storm are caused by atmospheric conditions in that region. (Salmon, 1989: 47)

The barometer is among the least controversial examples of a non-explanation in the literature. Now, if the barometer reading is not an explanation of the storm at all, then it is $a$ fortiori not a causal explanation of the storm. Indeed, the very fact that the barometer reading does not seem to provide for a causal explanation is often taken to lend plausibility to the idea that explanations must be causal.

However, notice that the information that the barometer drops sharply does rule out certain possible causes of the storm. It rules out all those possible causes of the storm that would also cause that barometer to change in any other way, e.g. by rising or keeping steady. Thus the barometer reading is a body of fact about what causes, if any, the storm had. Incidentally, the "explanans" in the barometer example also includes "a body of fact about what it would have taken for some specific alternative or range of alternatives to $\mathrm{E}$ to have occurred instead." This is because the "explanans" in the example includes a law governing the correlation between barometer readings and weather conditions, which rules out many 
possible causal histories of the various specific alternatives to the storm. For example, it rules out that clear weather (a specific alternative to the storm) is caused by something that also causes the barometer to drop sharply.

It might be objected that this shows only that the barometer counts as a partial causal explanation on STCE. That is true, but irrelevant. The uncontroversial judgment about the barometer is not that the barometer reading provides only a partial explanation of the storm it is that it doesn't explain the storm at all. The barometer reading is simply not explanatorily relevant to the storm, so the storm is not even partially explained by it. Thus it follows that the barometer reading a fortiori doesn't causally explain the storm even partially, contrary to what STGE entails. Hence STGE is false.

It might also be objected that although STCE is false, it does not matter much for Skow's overall defense of the thesis that all explanations are casual. Perhaps the way in which Skow argues against the putative counterexamples does not rely on this part of his theory of causal explanations? To see why that's not so, consider the class of putative counterexamples that most of Skow's paper is concerned with, viz. explanations that merely cite laws of nature. Here is an example that is closely analogous to one discussed by Skow: "That particle is traveling slower than light because no material thing can travel faster than (or at) the speed of light." As Skow notes, this appears to be a non-causal explanation since the explanans doesn't cite any causes of the particle travelling under the speed of light. Skow's response is that the explanation is causal after all, because:

The [particle] explanation cites no cause of the [particle's] subluminal velocity, but does rule out causal histories in which [particles] left alone always accelerate to a superluminal speed and something special happened to prevent this one from doing so. (Skow, 2014: 455) 
As we have seen, however, ruling out some causal histories of an event is not sufficient for something to count as even a partial causal explanation of that event. If that were so, then the barometer reading would count as a partial causal explanation of the storm. So Skow's response to this case exploits the very part of his theory of causal explanations that the barometer explanation shows to be mistaken. Put differently, the objection here presented against Skow's theory is an objection to precisely that part of the theory he uses to argue against an important class of putative counterexamples.

Now, although I have put my objection to Skow's theory in terms of the barometer explanation, it should be noted that there is an abundance of information that would count as partially causally explanatory on STCE even though no one would ever dream of considering it causal explanatory. For example, the fact that I am having coffee right now rules out certain causal histories of the fact that I am sitting in front of a computer, viz. those causal histories in which the causes of me sitting down also cause me not to have coffee. Yet I clearly have not even partially causally explained why I am sitting in front of a computer by citing the fact that I am having coffee. The general point here is that something may rule out causal histories, either of the explanandum event or of the specific alternatives to that event (or both, as in the barometer example), without causally explaining the event even partially. Causal explanations, even when merely partial, must do more than rule out causal histories.

\section{A Modest Revision of Skow's Theory}

I have argued that Skow's theory of causal explanation is overly permissive and thus cannot be relied upon in a defense of the thesis that all explanations are causal. Of course, this does not show that Skow's thesis is false, only that Skow's defense of it fails. Those seeking to defend the thesis might respond by attempting to construct another theory of causal explanation and argue that this new theory counts all explanations as causal. In order for such a defense to 
succeed, the new theory would have to be at least as plausible as alternative theories of causal explanation on which there are non-causal explanations. This section provides a reason for pessimism about this project by sketching what I take to be a plausible (but still very permissive) theory of causal explanation on which there are non-causal explanations.

As a point of departure I will take Skow's own theory of causal explanations, SKCE, and modify it in light of the problem identified in the previous section. Recall that the problem with Skow's theory is that it counts non-explanations such as the barometer as (partial) causal explanations. Now, since the "explanans" in the barometer explanation does rule out certain causal histories of the storm, it seems that the mere ruling out of some causal histories of $\mathrm{E}$ should not count as giving even a partial causal explanation of $\mathrm{E}$. The moral of the story is thus that merely ruling out causal histories does not by itself make some piece of information partially causally explanatory. However, it is also clear that a partial causal explanation of E needn't specify the entire causal history of E, so in what sense would a causal explanation be partial if not by merely ruling out some causal histories?

We may begin to answer this question by considering the fact that explanations are answers to certain questions - what Salmon (1989) calls explanation-seeking why-questions. So what is it to give a partial answer to a question? If my spouse asks "What's for dinner?", I might partially answer the question by saying "Not pizza", thereby ruling out certain possible dinners. Another way for me to partially answer my spouse's question is to specify part of what's for dinner, e.g. by saying "Well, there will be potatoes". At least in certain conversational contexts, it will be understood that we will not merely be having potatoes for dinner, although potatoes will be included. The point here is that the second answer works not only by ruling out certain dinners (such as potato-free pizza) but also by specifying part of what the dinner consists in. While the first answer partly specifies what's for dinner, the second answer specifies part of what's for dinner. 
Now, the barometer is an example in which the explanans partly specifies the causal history of the explanandum, for it rules out causal histories in which the storm's cause also causes a rise in the barometer, for example. Yet the explanans intuitively does not specify part of the explanandum's causal history, since it fails to specify any event or sequence of events in the storm's causal history (or, indeed, whether the storm has a causal history at all). In sum, although the drop of the barometer partly specifies the storm's causal history, it does not specify any part of it. This suggests that we can avoid the problem identified in the previous section by modifying Skow's theory of partial causal explanations as follows:

MTCE: A body of fact partially causally explains $\mathrm{E}$ iff it is a body of fact that specifies some part of the causal history of E; or it is a body of fact that specifies some part of the casual history that would have had to occur in order for some specific alternative or range of alternatives to $\mathrm{E}$ to have occurred instead.

To clarify, MTCE counts as partially causally explanatory any specification of an event or sequence of events in either the actual causal history of the explanandum event or the counterfactual causal history of an alternative or range of alternatives to the explanandum event. Here, "causal history" is taken in a broad sense that includes the possibility that the lack of a cause at some particular point may count as a part of an event's causal history. Thus, the fact that something is uncaused would count as causally explanatory on MTCE. 4,5

${ }^{4}$ To be clear, I am not defending MTCE here as an adequate account of partially causal explanations. Indeed, in my view MTCE is still overly permissive since it counts information that specifies any part of some event's causal history as a partial causal explanation of that event. So, if the Big Bang is a part of all actual causal histories, then the fact that the Big Bang happened counts as a partial causal explanation of all actual events. Also, depending on one's intuitions and how one individuates events, MTSC might also be too restrictive. To see this, consider the following case 
(suggested to me by Brad Skow in personal communication): Suppose Jones has a black eye because Smith punched him in the face. It might be thought that the information that someone punched Jones in the face suffices to partially causally explain Jones's black eye, and yet being punched by someone in the face arguably does not specify any particular cause of the black eye. (It does not specify whether the puncher was Smith, or Brown, or someone else.) However, if one wanted to defend MTCE, one could point out that the information that Jones was punched in the face by someone does specify that the causal history of Jones's black eye included a punch. If one adopts criteria for individuation of events such that punching counts as a distinct event (over and above Smith's punching, Brown's punching, etc.) this would count as partially causally explanatory on MTCE.

${ }^{5}$ It should be noted that this theory does conflict with an example that Skow takes to be of a partial causal explanation. Skow (2014: 448) says that the fact that Huey did not throw the rock at the window partially causally explains why the window broke since it rules out one particular cause of the window breaking and narrows down the list of possible causes to Dewey and Louie (who were the only other people around who might have thrown a rock at it). On MTCE, this is false since the information that Huey did not throw the rock does not specify any event or sequence of events in the causal history of the breaking of the window.

Although I am not defending MTCE here (see previous footnote), it does seem to me that MTCE gets this case right (and that Skow's original theory, STCE, gets it quite wrong). I cannot imagine a situation in which the piece of information that Huey did not break the window would even partially satisfy someone's request for a causal explanation of the fact that the window broke. (Of course, someone who is interested only in what Huey did - e.g. his parents - might be satisfied with this information, but such a person is not requesting an explanation of the fact that the window broke.) That said, I acknowledge that Huey throwing the rock at the window provides some information about what the partial causal explanation might be - thus we could say that it is causalexplanatory information relevant to the window breaking. But this is also true of the "explanans" in the barometer example, and so that cannot make Huey's non-throw into a partial causal explanation.

An anonymous reviewer points out that given the background knowledge that Huey, Dewey or Louie broke the window, the proposition that Louie broke the window is equivalent to the conjunctive proposition that neither Huey nor Dewey broke the window. So suppose Louie broke the window. Since the proposition that Huey did not break the window is one conjunct in a conjunction that is equivalent (given background knowledge) to this proposition about the actual cause of the breaking of the window, the reviewer suggests that one might think that Huey not breaking the 
While MTCE is admittedly only the beginning of a theory of causal explanations, it is precise enough to allow us to evaluate whether all explanations are causal on this theory. Consider the particle explanation from the previous section: "That particle is travelling slower than light because no material thing can travel faster than (or at) the speed of light." 6 The first thing to note about the example is that the particle travelling at the speed of light does have a causal history. On one possible causal history of the event, the particle just now came out of a particle accelerator - one that isn't powerful enough to accelerate the particle above a certain speed, which is lower than that of light. On another possible causal history, the particle was travelling slower than the speed of light a split-second ago - and since no forces have acted on the particle between now and then, it's still travelling slower than light. Both of these scenarios

window is a partial causal explanation. In response, I deny the assumption that the fact that some proposition is a conjunct in a conjunction that is equivalent (given background knowledge) to a proposition about the cause of some event suffices for the proposition to be a partial causal explanation of the event (although it may provide information that is of causal-explanatory relevance to the event). In fact, to my mind the failure of this assumption is adequately illustrated by the HueyDewey-Louie example, since the information about Huey's non-throw is intuitively not a partial causal explanation. For those who are not convinced by that case, here is another example: Suppose that victim $\mathrm{V}$ was murdered by suspect $\mathrm{S}$. Given the background knowledge that the murder was a one-man job performed by a human being, this is equivalent to a proposition of the form " $\mathrm{X}_{1}$ did not murder $\mathrm{V}$, and $\mathrm{X}_{2}$ did not murder $\mathrm{V} \ldots$ and $\mathrm{X}_{\mathrm{n}}$ did not murder $\mathrm{V}$ ", where $\left\{\mathrm{X}_{1}, \ldots, \mathrm{X}_{\mathrm{n}}\right\}$ is the set of all human beings except $\mathrm{S}$ at the time to the murder. However, the information that a random person did not murder $\mathrm{V}$ is surely not a partial causal explanation of V's death.

${ }^{6}$ In what follows, I will assume (as Skow himself does implicitly) that this is not an in-virtue-of explanation, i.e. an explanation in which the explanans grounds the explanandum. Admittedly, the notion of grounding is still being worked out in the metaphysics literature, so it is unclear what exactly will count as an in-virtue-of explanation. (See, for example, Schaffer (2009), Rosen (2010), and Audi (2012).) However, I have seen no suggestion in the literature that particular events may be grounded in the laws that govern them in the manner required for the event that the particle travels slower than light to be grounded in the law that nothing travels faster than (or at) the speed of light. 
are possible causal histories of the particle travelling under the speed of light - and they are contrary causal histories since they disagree vis-à-vis how things stood in the world a splitsecond ago.

Now, note that the information that no material thing can travel faster than (or at) the speed of light does not specify which of these possible causal histories is correct about the part of the actual causal history that took place a split-second ago. Thus the part of the causal history of the particle's travelling slower than the speed of light that took place a split-second ago are not specified by the explanans. The same is clearly true of any other parts of the particle's causal history, so the explanans does not specify any part of that causal history. Neither does the explanans specify any part of what it would have taken for some specific alternatives to the explanandum to have occurred instead. In this case, a specific alternative to the explanandum would be an event in which the particle travelled at or above the speed of light. To be sure, Skow is right to point out that the explanans tells us something about the causal histories of such events, viz. that "faster-than-light motion would have required different physical laws." (Skow, 2014: 456) However, this clearly doesn't suffice to specify any part of the causal histories of the particle travelling at a given superluminal speed - at best, it merely rules out certain causal histories of these alternative events.

In sum, then, the explanans in the particle explanation neither specifies some part of what causes, if any, the explanandum had; nor specifies some part of what it would have taken for some specific alternative or range of alternatives to the explanandum to have occurred instead. Hence the particle explanation is not even partially causal according to MTCE. This further undermines Skow's claim that all explanations are (even partially) causal, since MTCE is designed to be as close to STCE as possible without having the absurd consequence that the barometer and other uncontroversial non-explanations count as partial causal explanations. Of course, since MTCE is not the only possible alternative to STCE, this does not conclusively 
show that there are non-causal explanations. However, it does show that those who seek to argue otherwise must not only present a theory of causal explanation on which there are no non-causal explanations, but also show why such a theory should be preferred to MTCE. Since MTCE is both plausible and still very permissive, we should be pessimistic about the prospects for finding such a theory.

\section{Conclusion}

I have undermined Skow's defense of the thesis that there are no non-causal explanations (of particular events, excluding in-virtue-of explanations, and where "non-causal" is understood as meaning not even partially causal). Skow's defense of this thesis against putative counterexamples turns out to rest on a theory of partial causal explanations that I have argued is overly permissive. I also presented a slightly less permissive theory in the same spirit, and argued that one class of counterexamples still holds up on this theory. Since this modified theory is still quite permissive, I conclude that the prospects for arguing that all explanations are causal using some alternative to Skow's theory are dim. At the very least, it is clear that a plausible theory of causal explanations on which all explanations of the requisite kind are even partially causal is yet to be found. ${ }^{7}$

\footnotetext{
${ }^{7}$ I would like to thank Michael Bertrand, Marc Lange, Bradford Skow, and two anonymous reviewers for this journal for helpful comments on drafts of this paper. I would also like to thank those who participated in the reading group on non-causal explanations in the Spring of 2014 at the University of North Carolina at Chapel Hill for valuable discussions on these issues.
} 


\section{References}

Audi, P. (2012). "Grounding: Toward a Theory of the In-virtue-of Relation", Journal of Philosophy 109: 685-711.

Hempel, G. G. (1965). Aspects of Scientific Explanation and Other Essays in the Philosophy of Science. New York, NY: The Free Press.

Lewis, D. K. (1986a). "Causal Explanation”, in his Philosophical Papers: Volume II (New York, NY: Oxford University Press), pp. 214-40.

Nerlich, G. (1979). "What Can Geometry Explain?”. The British Fournal for the Philosophy of Science 30: 69-83.

Rosen, Gideon (2010). "Metaphysical Dependence: Grounding and Reduction," in B. Hale and A. Hoffmann (eds.), Modality: Metaphysics, Logic, and Epistemology (Oxford: Oxford University Press), pp. 109-36.

Salmon, W. G. (1984). Scientific Explanation and the Causal Structure of the World. Princeton, NJ: Princeton University Press.

Salmon, W. C. (1989). "Four Decades of Scientific Explanation", in P. Kitcher and W. C. Salmon (eds.), Scientific Explanation (Minneapolis, MI: University of Minnesota Press), pp. 3-219.

Schaffer, Jonathan (2009). "On What Grounds What," in D. Chalmers, D. Manley and R. Wasserman (eds.), Metametaphysics (Oxford: Oxford University Press), 347-83.

Skow, B. (2014). "Are There Non-Causal Explanations (of Particular Events)". The British Fournal for the Philosophy of Science 65: 445-467. 